\title{
Optimization of Reactive Power Compensation Based on Fuzzy Mathematics
}

\author{
Dawa Lunzhu ${ }^{1}$, Tao Kui ${ }^{1}$, Ni Ping ${ }^{1}$, Basang Dunzhu ${ }^{1}$, Dong Zhihua ${ }^{1}$, Xu Feng and Pan Zhiyuan ${ }^{2 *}$ \\ ${ }^{1}$ State Grid Lhasa Power Supply Company, 24-4\# Niang Re Road, Lhasa,850000， China \\ ${ }^{2}$ State Grid of China Technology College, 500\# Er Huan Nan Road, Jinan, 250002, China
}

\begin{abstract}
This paper proposes a heuristic method for optimization of reactive power compensation. Firstly, two fuzzy sets of node voltage and cost savings are formed. The compensation location with the greatest compensation suitability is obtained by fuzzy reasoning. Considering the operation modes of several different loads, the mathematical model of reactive power compensation optimization is established, and fuzzy multi-objective optimization is adopted. The method solves the fixed and variable compensation capacity, and calculates the compensation capacity for each existing compensation position cycle until the change does not occur. The above process is repeated until the new compensation position is no longer saved, and finally all the compensation positions, capacity and total savings can be obtained. The example analysis shows the effectiveness and practicability of the method.
\end{abstract}

\section{Introduction}

The reactive power compensation of the distribution network can determine the position, type and capacity of the reactive power compensation equipment. Under the constraints of different load operation modes, it can maximize the difference between the cost of energy loss reduction and the investment cost of reactive power compensation equipment. It also can reduce losses at peak loads, improves voltage and power factor, increase feeder power and substation capacity.

Reactive power compensation is a multi-objective mixed integer optimization programming problem. The analytical method [1] uses the $2 / 3$ rule to determine the compensation position and capacity, but the compensation position usually corresponds to the actual node, and the compensation capacity is also a rough estimate. According to the principle of decomposition coordination, the literature [2-5] decomposes the reactive power compensation into the maximum, minimum and general load modes, and unifies it to the maximum load mode, which is solved by the gradient method of nonlinear programming, However, it is easy to fall into the local optimal solution for non-convex problems, and no method for determining the compensation position is given. The literature [6,7] uses the sensitivity-based heuristic method to determine the control and configuration of the reactive power supply, and then applies the Bender's decomposition method to decompose the problem into the investment sub-problem and the running sub-problem. The linear programming method is used to solve the problem. The algorithm needs to first determine the compensation position. The compensation capacity and the number of units are calculated again, but the number of compensation positions is manually intervened. The genetic algorithm [8-15] usually determines the compensation position by the sensitivity analysis method, but the number of compensation positions needs to be manually intervened. Although the genetic algorithm guarantees the optimal solution effect, the number of different compensation positions is different. In [16], we first find the branch that causes the maximum loss due to reactive current, and then take the node that contributes the largest reactive current of the branch as the compensation node, and calculate the node compensation by the difference between the total energy reduction cost of the system and the capacitor investment cost. Capacity, repeat the above process until the new compensation node no longer saves money. In [17], the method of determining the compensation position in [16] is improved. The node with the largest reactive current drop loss is used as the compensation node. The other processes are the same as in [16]. In [18], each node is compensated for the reactive power equal to the reactive load of the node, and the network loss is calculated. The network loss after compensation and the node voltage before compensation are respectively composed of fuzzy sets, and the maximum degree of suitability is obtained by fuzzy reasoning. The compensation node, the other process is the same as the literature [16], the network loss change and the node voltage are comprehensively considered when determining the compensation position. The advantage of the literature [16-18] is that the calculation amount is small, there is no convergence problem, but the compensation position is determined without considering the change of the post-compensation cost, it is difficult to obtain the maximum cost savings, and the

*Corresponding author's e-mail: z.y.pan@qq.com 
mutual influence of the capacity of each compensation node has been not considered, so it is easy to fall into the local optimal solution.

Based on the above, based on the two indicators of cost and voltage, this paper uses fuzzy reasoning method to determine a node with the greatest compensation suitability, and considers the benefit and voltage of compensation. The fuzzy multi-objective optimization method is used to calculate the fixed and variable nodes' compensation capacity. And cyclically calculate the capacity of each known compensation position to avoid falling into the local optimal solution. Repeat the above process until the new compensation position is no longer cost-saving, and there is no need to manually intervene to compensate for the number of positions selected.

\section{Position of reactive compensation}

The phase angle of the load node voltage of the distribution network is generally small, which can be approximately equal to zero, and the node voltage is close to the rated value after compensation. The change of reactive power mainly affects the reactive current, and the influence on the active current can be neglected. Therefore, according to the node injection Savings after reactive current and pre-compensation voltage inference compensation position.

The calculation method of cost saving after compensating reactive current of radial distribution network is given as follows: for a radial distribution network with $n$ nodes of $b$ branches, assume that reactive current $J_{m c}$ is compensated at node $m$ and reactive current $I_{j}^{\text {new }}$ of branch $j$ is:

$$
I_{j}^{\text {new }}=I_{j}+D_{j m} J_{m c}
$$

Where, $I_{j}$ is the reactive current compensating the front branch $j ; D=A^{-1}, \quad A$ is the node branch incidence matrix.

The cost savings $S_{E}$ can be expressed as:

$$
S_{E}=\sum_{j=1}^{b}\left[\left(I_{j}\right)^{2}-\left(I_{j}+D_{j m} J_{m c}\right)^{2}\right] K_{E} R_{j}-K_{I} J_{m c}
$$

Where, $R_{j}$ is the resistance of the branch $j ; b$ is the number of branches; $K_{E}$ is the electricity price $(¥ / \mathrm{kWh}) ; K_{I}$ is the unit reactive current investment fee (¥/kA). The unit reactive current investment cost can be approximately equal to the unit cost of the reactive power compensation equipment multiplied by the rated voltage.

When $S_{E}$ is maximum:

$$
\frac{\partial S_{E}}{\partial J_{m c}}=0
$$

Combining equations (2) and (3):

$$
J_{m c}=\frac{-K_{I} / K_{E}-\sum_{j=1}^{b} 2 I_{j} D_{j m} R_{j}}{\sum_{j=1}^{b}\left(D_{j m}\right)^{2} R_{j}}
$$

Substituting equation (4) into equation (2) can get the maximum cost savings after compensating reactive current of the node $m$. The steps for determining the reactive compensation node by fuzzy inference are given below:

1) Calculate the cost savings for each node, and select the maximum value, then divide the savings cost of all nodes by the maximum value, so that the cost savings are in the interval $[0,1]$, which constitutes a fuzzy set of cost savings;

2) The value of the node voltage before compensation is composed of the node voltage fuzzy set;

3) The fuzzy inference method of the literature [18] is used to determine the reactive power compensation node, and attention is paid to replace the fuzzy set with reduced network loss into a cost-saving fuzzy set.

The maximum cost savings and voltages of the reactive currents at each node compensate for the fuzzy set. Compared to the literature [8, 15], the cost savings are more and the improvement of the node voltage is better.

\section{Capacity of reactive power compensation}

The objective function of capacity of reactive power compensation is :

$S_{E \max }=K_{E} \Delta E_{\max }-K_{c} Q_{c}$

$S_{\text {Enor }}=K_{E} \Delta E_{\text {nor }}-K_{c} Q_{c}$

$S_{E \min }=K_{E} \Delta E_{\min }-K_{c} Q_{c}-K_{l} Q_{l}$

$S_{\text {peak }}=\Delta P_{\text {peak }}$

s.t. $\mathrm{V}_{\min } \leq \mathrm{V} \leq \mathrm{V}_{\max }$

Where, $S_{E \max }, S_{E m o r}, S_{E \min }$ are the difference between the energy loss saved under maximum load, general load and minimum load and the investment cost of compensation equipment respectively. $\Delta E_{\max }, \Delta E_{\text {mor }}, \Delta E_{\min }$ are the power loss saved under maximum load, general load and minimum load respectively. $S_{\text {peak }}, \Delta P_{\text {peak }}$ are losses reduced under peak load; $Q_{c}, Q_{l}$ are capacitive reactive power and inductive reactive power compensation capacity respectively. $K_{c}, K_{l}$ are the unit investment cost of capacitor and reactor respectively( $¥ /$ kvar). $\mathrm{V}_{\text {min }} \leq \mathrm{V} \leq \mathrm{V}_{\max }$ is constraint of node voltage.

Inductive reactive compensation in equation (5) is only considered under the minimum load mode before capacitive reactive compensation. If there is a node with a voltage limit, we should adjust the ratio of the 
transformer connected to the node at the first, when the ratio is adjusted to the upper limit, when the voltage is still limited, consider the inductive reactive compensation.

The multi-objective fuzzy optimization method is used to solve equation (5). The membership function of each target is expressed as follows:

$$
u_{s}\left(Q_{c}\right)=\left\{\begin{array}{cc}
1 & S\left(Q_{c}\right)>M_{s} \\
\frac{S\left(Q_{c}\right)-m_{s}}{M_{s}-m_{s}} & m_{s} \leq S\left(Q_{c}\right) \leq M_{s} \\
0 & S\left(Q_{c}\right)<m_{s}
\end{array}\right.
$$

Where, $m_{s}=\min S\left(Q_{c}\right) ; M_{s}=\max S\left(Q_{c}\right)$.

Each objective function in equation (5) has a membership function as shown in equation (6). For each membership function, when $m_{s}=0, M_{s}$ can be solved by the following methods:

$$
\frac{\partial S\left(Q_{c}\right)}{\partial Q_{c}}=0
$$

The membership function of equation (6) is shown in figure 1:

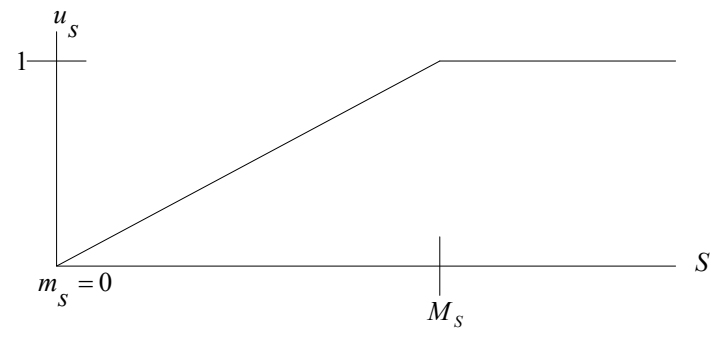

Figure 1. Membership function of cost objective.

The $Q_{c \text { min }}$ in the load mode with the smallest compensation capacity can be used as the fixed compensation. The membership function $u_{s}\left(Q_{c}\right)$ in the load mode with the largest compensation capacity and the membership function $u_{\text {speak }}\left(Q_{c}\right)$ at the peak load constitute the objective function:

$$
\begin{aligned}
& \max u_{c}=u_{s} \times u_{\text {speak }} \\
& \text { s.t. } \quad \mathrm{V} \leq \mathrm{V}_{\max }
\end{aligned}
$$

The $Q_{c}$ obtained from the above equation includes the fixed capacitor capacity $Q_{c \text { min }}$ and the variable capacitor capacity $Q_{c}-Q_{c \min }$.

The method of solving the equation (9) is based on the fixed capacitor capacity $Q_{c \text { min }}$, the capacity $\Delta Q_{c}$ of each group of the variable capacitor group is stepwise, gradually increasing the compensation capacity, and calculating $u_{c}$ until the $u_{s \max }$ or $u_{\text {speak }}$ has a negative value. The $Q_{c}$ that takes the $u_{c}$ maximum and satisfies the voltage constraint is used as the compensation capacity.

The cost savings $S_{\text {total }}$ is:

$$
S_{\text {total }}=K_{E}\left(\Delta E_{\max }+\Delta E_{\text {nor }}+\Delta E_{\min }\right)-K_{c 1} Q_{c \min }-K_{c 2}\left(Q_{c}-Q_{c \min }\right)
$$

Where, $K_{c 1}$ is the unit cost of fixed capacitor investment; $K_{c 2}$ is variable capacitor investment unit cost.

In the equation (5), if the peak load network loss is not considered, the $Q_{c \text { min }}$ in the load mode with the smallest compensation capacity can be used as the fixed compensation capacity, and the difference between the $Q_{c \max }$ and the $Q_{c \min }$ in the load mode with the largest compensation capacity is used as the variable compensation capacity. The total cost saved in this way is greater than that of equation (9), but the effect of loss and voltage increase at peak load is not obvious. The following example shows that considering the peak load network loss has little effect on the total cost saved, but it can be effective reduce losses at peak load and improve node voltage.

\section{Algorithm steps}

1) In the maximum load mode, the cost is saved after each node is injected with reactive current according to equations (4) and (2);

2) The cost savings and the voltages of the respective nodes before compensation are respectively composed of fuzzy sets, and the fuzzy inference method of the literature [18] is used to determine the node with the greatest compensation suitability;

3) Calculate the compensation capacity of the node under three load modes separately, take the minimum compensation capacity as the fixed compensation, calculate the variable compensation capacity according to equation (8), and calculate the cost savings $S_{\text {total }}$ after the node compensation according to equation (9). If $S_{\text {total }} \leq 0$, the operation ends.

4) If there are $M(M>1)$ nodes that have reactive power compensation, for node $i=1$, if the compensation capacity of other nodes is unchanged, recalculate the compensation capacity according to step 3 . If the compensation capacity calculated twice before and after this node does not change, go to step 6 .

5) Repeat step 4 for the node $i=2, \ldots M$ of the compensated capacitor.

6) Accumulate the cost saved by each compensation node compensation capacitor, and accumulate the compensation capacity under different load operation modes into the system, and go to step 1;

The algorithm considers the interaction of the existing capacity between the various compensation nodes and the impact on the new compensation points. The latter example shows that steps 4) and 5) have quadratic convergence and no oscillation occurs. It is well converged after three to four calculations. 


\section{Case studies}

A heavy-duty, multi-branch feeder of an actual distribution network is shown in figure 1. It consists of 42 nodes and 41 branches. The reference capacity is $100 \mathrm{MVA}$, the rated voltage is $11 \mathrm{KV}$, and the cost coefficient per unit of electricity is $0.5 ¥ / \mathrm{kWh}$, the planning period is 10 years, the capacity of each group of variable capacitors is $10 \mathrm{kvar}$, the total investment of capacitors is $20 ¥ / \mathrm{kvar}$, and the investment cost of reactive current is approximately $200 ¥ / \mathrm{A}$.

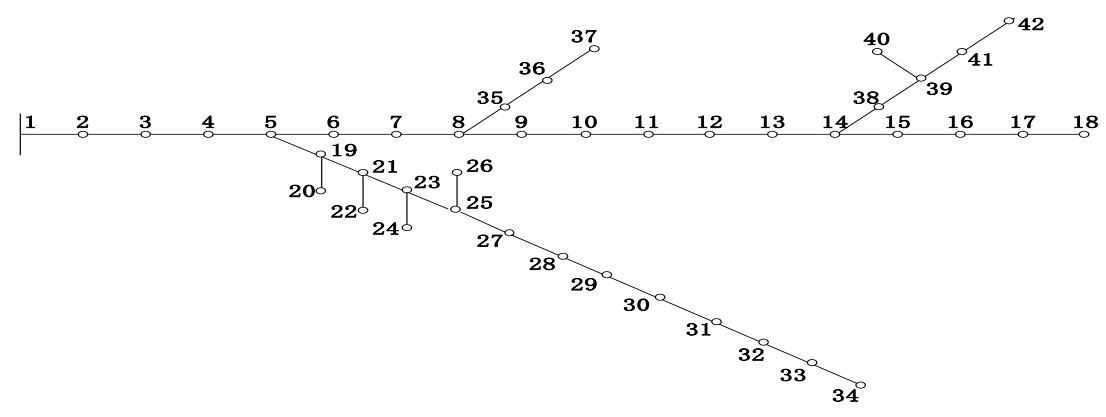

Figure 2. Diagram of 42-bus distribution system.

Table 1. Allocation node.

\begin{tabular}{c|c|c|c}
\hline Compensation node & Cost membership & Voltage membership & Configuration suitability \\
\hline 12 & 1 & 0.913811 & 0.916 \\
\hline 27 & 1 & 0.951647 & 0.75 \\
\hline
\end{tabular}

Table 1 is the result of fuzzy inference. The configuration of nodes with large cost membership is also suitable, which is consistent with the principle of saving cost as the main goal in the process of reactive power compensation.

Table 2. Allocation capacity and iteration process.

\begin{tabular}{c|c|c|c}
\hline Number of iterations & Number of node & Fixed capacitor capacity (Mvar) & Adjustable capacitor capacity (Mvar) \\
\hline \multirow{2}{*}{1} & 12 & 1.19 & 0.78 \\
\cline { 2 - 4 } & 27 & 0.76 & 0.97 \\
\hline \multirow{2}{*}{2} & 12 & 0.96 & 0.67 \\
\cline { 2 - 4 } & 27 & 0.88 & 0.9 \\
\hline \multirow{2}{*}{3} & 12 & 0.93 & 0.67 \\
\cline { 2 - 4 } & 27 & 0.9 & 0.89 \\
\hline
\end{tabular}

Table 2 is an iterative process of the mutual influence of the existing compensation nodes. The first iteration result and the third iteration result are very different, indicating that there is a large influence between the compensation capacities of each node, and it is compensated by three iterations. The capacity does not change and the algorithm has good convergence.

Table 3. Comparison of lowest voltage before and after capacitor allocation.

\begin{tabular}{c|c|c|c|c}
\hline & Peak load $(\mathrm{pu})$ & Maximum load $(\mathrm{pu})$ & General load $(\mathrm{pu})$ & Minimum load (pu) \\
\hline Before the compensation & 0.851 & 0.906 & 0.914 & 0.924 \\
\hline After the compensation & 0.899 & 0.936 & 0.941 & 0.948 \\
\hline
\end{tabular}

Table 3 compares the minimum node voltages before and after compensation. In the maximum, normal, and minimum load modes, the node voltage is calculated under the maximum cost savings, and the compensated voltage is significantly improved.

Table 4. Comparison of different objective function.

\begin{tabular}{l|c|c|c}
\hline & $\begin{array}{c}\text { Total cost saving (ten } \\
\text { thousand yuan) }\end{array}$ & $\begin{array}{c}\text { Minimum node voltage } \\
(\mathrm{pu})\end{array}$ & $\begin{array}{c}\text { Loss reduction } \\
(\mathrm{kW})\end{array}$ \\
\hline Peak load is not considered & 189.6 & 0.885 & 502.9 \\
\hline Peak load is considered & 187.36 & 0.899 & 475.3 \\
\hline
\end{tabular}


It can be seen from Table 4 that the total cost of considering and not considering the peak load is not much different, but considering the peak voltage, the node voltage and the loss reduction effect are significantly improved.

Table 5. Comparison of determination of location.

\begin{tabular}{c|c|c|c|c}
\hline & $\begin{array}{c}\text { Total cost saving } \\
\text { (ten thousand yuan) }\end{array}$ & $\begin{array}{c}\text { Minimum node voltage } \\
\text { at maximum load (pu) }\end{array}$ & $\begin{array}{c}\text { The first } \\
\text { compensation node }\end{array}$ & $\begin{array}{c}\text { The second } \\
\text { compensation node }\end{array}$ \\
\hline Proposed method & 187.36 & 0.936 & 12 & 27 \\
\hline Method in literature [8] & 184.7 & 0.935 & 11 & 27 \\
\hline Method in literature [15] & 184.2 & 0.935 & 42 & 32 \\
\hline
\end{tabular}

Table 5 shows the compensation position by different methods. The comparison of the compensation capacity is calculated by the method in this paper. It can be seen from the table that the method of determining the compensation position method has the largest cost and the node voltage improvement effect is the best.

\section{Conclusion}

(1) The fuzzy set is formed by saving cost and voltage respectively, and the node with the greatest compensation suitability is obtained as the compensation node by fuzzy inference. Taking into account the two indicators of cost savings and voltage, the determined compensation nodes can maximize cost savings and improve voltage.

(2) In addition to considering the saved energy loss cost and reactive power compensation equipment investment, the mathematical model also takes into account the peak loss effect, avoiding excessive loss and high voltage at peak load.

(3) The capacity of the load mode with the smallest compensation capacity is determined as fixed compensation, and the variable compensation is calculated according to the load mode with the largest compensation capacity and the peak load, so that the system operation mode is more flexible.

(4) Although the heuristic method is adopted, each time a compensation point and compensation capacity are determined, the compensation capacity is calculated cyclically for each existing compensation point, and the mutual influence between the capacities of each compensation point is fully considered to avoid falling into the local optimal solution.

\section{Reference}

1. Y.G. Bae. Analytical method of capacitor allocation on distribution primary feeders[J]. IEEE Trans. on Power Apparatus and Systems, 1978, 97(11):1232-1238.

2. Qiu J, Shahidephour S.M. A new approach for minimizing power losses and improving voltage profile $[\mathrm{J}]$. IEEE Trans. on Power System, 1987,2(2):287-295.

3. Li Lincuan, Wang Jianyong, Chen Liyi, Song Wennan. Optimal reactive power planning of electrical power system[J]. Proceedings of the CSEE, 1999, 19(2): 66-69.

4. M.E. Baran, F.F. Wu. Optimal capacitor placement on radial distribution systems[J]. IEEE Trans. on Power System, 1989,4(1):725-734.

5. M.E. Baran, F.F. Wu. Optimal sizing of capacitor placed on a radial distribution systems[J]. IEEE Trans. on Power System, 1989,4(1):725-734.

6. Wang Chenshan, Tang Xiaoli, Yu Yixin, et al. Application of benders decomposition based on a hierarchical programming in reactive power planning $[\mathrm{J}]$. Automation of Electric Power Systems, 1998, 22(11):14-17.

7. Gao Huimin, Zhang Jianmin, Jiang Li. Optimal Location of reactive power compensation for distribution network based on second order loss-reactive power sensitivity matrix. Power System Technology, 2014, 38(7): 1979-1983.

8. Ma Xiufan, Cui Huanjun. An improved genetic algorithm for distribution network planning with distributed generation[J]. Transactions of China Electrotechnical Society, 2011, 26 (3) : 175-181.

9. Zhang Lizi, Shu Jun, Lin Xiaoshu, et al. Reactive power planning based on genetic algorithm[J]. Proceedings of the CSEE, 2000, 20(6): 5-8

10. Zhang Yongjun, Ren zhen, Zhong Hongmei, et al. Cataclysmic genetic algorithms based on optimal reactive power planning $[\mathrm{J}]$. Automation of Electric Power Systems, 2002, 26(23): 29-32.

11. Yun Xinmei, Li Yan, Xiong Xinyin, et al. Optimal shunt capacitor placement using particle swarm optimization algorithm with harmonic distortion consideration[J]. Proceedings of the CSEE, 2003, 23(2): 26-30.

12. Hu Haiyan, Wu xiaomeng, Li Jian. Genetic algorithm based on optimal dynamic planning of low-voltage side reactive power compensators[J]. Electric Power Automation Equipment, 2005, 25(3): 26-29.

13. Han Xiaojuan, Wang Lina, Gao Tong, et al. Generation planning of grid-connected micro-grid system with PV and batteries storage system based on cost and benefit analysis[J]. Transactions of China Electrotechnical Society, 2016, 31(14): 31-39. 
14. Ding Xiaoqun, Wang Kuan, Shen Maoya, et al. Application of Genetic Algorithm combining modal analysis in reactive power planning for distribution network[J]. Power System Technology, 2006, 30(17): 47-50.

15. Xu Yuqin, Liu Yang, Xie Qing. Research on reactive power planning of distribution network based on life cycle cost $[\mathrm{J}]$. Power System Protection and Control, 2018, 46(11): 30-36.

16. T.S. Abdel-Salam, A.Y. Chikhani, R. Hackam. A new technique for loss reduction using compensating capacitors applied to distribution systems with varying load condition[J]. IEEE Trans. On Power Delivery, 1994, 9(2): 819-827.

17. M. Chis, M.M.A. Salama, S. Jayaram. Capacitor placement in distribution systems using heuristic search strategies[J]. IEE Preceeding Generation, Transmission and Distribution, 1997, 144(2): 225-230.

18. H.N.Ng, M.M.A. Salama, A.Y. Chikhani. Capacitor allocation by approximate reasoning: fuzzy capacitor placement[J]. IEEE Trans On Power Delivery, 2000, 15(1): 393-398. 